Ethics Approval Mice were maintained under pathogen-free conditions in accordance with the Association for Assessment and Accreditation of Laboratory Animal Care guidelines. All animal studies were approved by the NIH Intramural Animal Care and Use Committee under protocol LTIB-038. Consent N/A

http://dx.doi.org/10.1136/jitc-2020-SITC2020.0445

\section{IMMUNOPEPTIDOME CHANGES MEDIATED BY A NOVEL ERAP1 INHIBITOR LEADS TO TUMOR GROWTH INHIBITION}

${ }^{1}$ Peter Joyce ${ }^{1}$ Lesley Young* ${ }^{1}$ Martin Quibell, ${ }^{2}$ Jason Shiers, ${ }^{2}$ Carmen Tong ${ }^{2}$ Kristopher Clark, ${ }^{3}$ Edd James, ${ }^{3}$ Emma Reeves, ${ }^{4}$ Alihussein Remtulla, ${ }^{4}$ Henry Leonard, ${ }^{5}$ Camila de Almeida, ${ }^{3}$ Elisa Lori, ${ }^{6}$ Nicola Ternette, ${ }^{6}$ Fergus Poynton, ${ }^{1}$ Andrew Leishman. ${ }^{1}$ Grey Wolf Therapeutics Ltd, Oxford, UK; ${ }^{2}$ Sygnature Discovery, Nottingham, UK; ${ }^{3}$ University of Southampton, Southampton, UK; ${ }^{4}$ Charles River Laboratories, Portishead, UK; ${ }^{5}$ Certara, Chester, UK; ${ }^{6}$ University of Oxford, Oxford, UK

Background Clinical data demonstrates increased antigen presentation diversity is a key factor in determining response rates to checkpoint inhibitors. ${ }^{1}$ In addition to tumour mutational burden/microsatellite instability, increased HLA heterozygosity and HLA evolutionary diversity are non-overlapping factors recently identified to further diversify the immunopeptidome and improve clinical response to checkpoint therapies. ${ }^{2}$ 3 Endoplasmic reticulum aminopeptidase 1 (ERAP1) is an enzyme that trims peptides loaded into classical and nonclassical class I MHC molecules. ${ }^{4}{ }^{5}$ Ablation of mouse ERAAP modifies the immunopeptidome, resulting in improved immunogenicity, generation of CD8 T cell responses and tumor growth inhibition. ${ }^{6} 7$ Recently identified selective small molecules potently inhibit ERAP1 across key species and haplotypes. ${ }^{8}$ We report the further profiling of lead candidate ERAP1 inhibitors in human primary $\mathrm{T}$ cell in vitro assays and in vivo tumor models in mice.

Methods Human cancer cell lines treated with ERAP1 inhibitors in vitro or in vivo in xenograft mouse models were assessed by immunopeptidomics 9 to profile peptide repertoire changes. Novel or upregulated peptides were also tested in human immunogenicity assays. FACS analysis of T cells stimulated with Tyrosinase mRNA transfected human dendritic cells \pm ERAP1 inhibition was to assess $\mathrm{T}$ cell repertoire changes. ERAP1 inhibitor and anti PD-1 mAb combination was assessed in syngeneic mouse tumor models to investigate tumour growth inhibition and PD end-points (e.g. IHC).

Results Analysis of human cervical, lung, colorectal and melanoma cell lines carrying distinct HLA haplotypes demonstrates a consistent and profound effect of ERAP1 inhibition on the immunopeptidome. Novel and upregulated cancer associated antigens identified in association with multiple different HLA$\mathrm{A}$ and $\mathrm{B}$ alleles stimulate IFN $\gamma$ production in primary naïve human $\mathrm{T}$ cell immunogenicity assays. The impact of ERAP1 inhibition on the $\mathrm{T}$ cell repertoire to the melanoma antigen tyrosinase is ongoing. The combination of ERAP1 inhibitor and anti PD-1 mAb led to significant tumor growth inhibition in the CT26 syngeneic mouse tumor model that correlated with increased infiltration of $\mathrm{T}$ cells to the tumor. Further PD end-points to be analysed include immune gene array and TCR Vbeta repertoire.

Conclusions Grey Wolf ERAP1 inhibitors significantly modify the immunopeptidome both in vitro and in vivo across a broad range of HLA and tumor types. Combination of these inhibitors with anti PD-1 leads to significant $\mathrm{T}$ cell infiltration and tumor growth inhibition. Thus, ERAP1 mediated modulation of the immunopeptidome has the potential to drive anti tumor $\mathrm{T}$ cell responses and be a transformative immunotherapy.

\section{REFERENCES}

1. Rizvi N, Hellmann MD, Snyder $A$, et al. Mutational landscape determines sensitivity to PD-1 blockade in non-small cell lung cancer. Science. 2015;348 (6230):124-128.

2. Chowell D, Morris LGT, Grigg CM, et al. Patient HLA class I genotype influences cancer response to checkpoint blockade immunotherapy. Science 2018;359 (6375):582-587.

3. Chowell D, Krishna C, Pierini F, et al. Evolutionary divergence of HLA class I genotype impacts efficacy of cancer immunotherapy. Nature Medicine 2019;25 (11):1715-1720.

4. Shastri N, Nagarajan N, Lind KC, et al. Monitoring peptide processing for $\mathrm{MHC}$ class I molecules in the endoplasmic reticulum. Curr Opin Immunol 2014; 26:123-127.

5. Mpakali A, Maben Z, Stern LJ, et al. Molecular pathways for antigenic peptide generation by ER aminopeptidase 1. Mol Immunol 2018; 13:50-57.

6. James $E$, Bailey I, Sugiyarto $G$, et al. Induction of protective antitumor immunity through attenuation of ERAAP function. J Immunol 2013;190(11):5839-5846.

7. Manguso RT, Pope HW, Zimmer MD, et al. In vivo CRISPR screening identifies Ptpn2 as a cancer immunotherapy target. Nature 2017;547(7664):413-418.

8. Leonard, H Remtulla A, Poynton F, et al. AACR Annual Meeting 2020.

9. Purcell AW, Ramarathinam SH, Ternette N. Mass spectrometry-based identification of MHC-bound peptides for immunopeptidomics. Nat Protoc 2019;14 (6):1687-1707.

http://dx.doi.org/10.1136/jitc-2020-SITC2020.0446

\section{VISTA TARGETING REMODELS THE TUMOR MICROENVIRONMENT TO OVERCOME ADAPTIVE RESISTANCE}

'Janet Lines*, ${ }^{1}$ Evelien Schaafsma, ${ }^{1}$ Walburga Croteau, ${ }^{1}$ Mohamed ElTanbouly, ${ }^{1}$ Elizabeth Nowak, ${ }^{1}$ Nicole Smits, ${ }^{1}$ Cecilia Webber, ${ }^{1}$ Dina Rabadi, ${ }^{2}$ Jie Deng, ${ }^{3}$ Chao Cheng, ${ }^{1}$ Randolph Noelle. 'Dartmouth College, Lebanon, NH, USA; ' UCLA, Los Angeles, CA, USA; ${ }^{3}$ Baylor College of Medicine, Houston, TX, USA

Background VISTA is a negative checkpoint regulator prominently expressed in the TME of a wide variety of cancers. In a preclinical model of colorectal cancer, monotherapy of small tumors $\left(40 \mathrm{~mm}^{3}\right)$ with anti-VISTA results in markedly slowed tumor growth. Mice bearing significantly larger tumors (600 $\mathrm{mm}^{3}$ ) are resistant to anti-PD-1 and anti-CTLA4 treatment and all mice die following treatment, indicating checkpoint resistance. Inclusion of anti-VISTA leads to complete rejection of $50 \%$ of tumors.

Methods The underlying therapeutic mechanisms of leading to enhanced anti-tumor immunity in both models was investigated by high-dimensional scRNAseq of the CD45+ immune infiltrate of tumors 10 days after treatment initiation.

Results In both modes, anti-VISTA treatment stimulated several pathways involving myeloid activation and antigen-presentation. Multi-spectral imaging of anti-VISTA treated tumors supported increased antigen presentation, and suppression assays showed that the myeloid infiltrate was less suppressive to $\mathrm{T}$ cells. Transcriptional analysis of tumor-specific CD8 T cells showed that anti-VISTA therapy induced $\mathrm{T}$ cell pathways highly distinct from the anti-exhaustion effects of anti-PD-1 therapy.

Conclusions These data document the unique and complementary impact of targeting VISTA in contrast to PD-1 and CTLA-4 in both the myeloid and T cell lineages. These mechanistic insights strongly support the use of anti-VISTA to 